\title{
Diversidad, especificidad de forófito y preferencias microambientales de líquenes cortícolas de un bosque subandino en la región Centro de Colombia
}

\section{Diversity, phorophyte specificity and microenvironmental preferences of corticolous lichens in a sub-Andean forest in the Centro region of Colombia}

\author{
Nicolás Zárate-Arias ${ }^{1}$, ๑ Miguel Moreno-Palacios ${ }^{1}$, ๑ Alfredo Torres-Benítez ${ }^{1,2, *}$ \\ ${ }^{1}$ Facultad de Ciencias Naturales y Matemáticas, Universidad de Ibagué, Ibagué, Colombia \\ ${ }^{2}$ Instituto de Farmacia, Facultad de Ciencias, Universidad Austral de Chile, Valdivia, Chile
}

\begin{abstract}
Resumen
El objetivo del estudio fue contribuir al conocimiento de la composición y la diversidad, la especificidad de forófito y las preferencias microambientales de líquenes cortícolas de un bosque subandino en la región Centro de Colombia (Ibagué, Tolima). Se seleccionaron 25 individuos de cinco especies arbóreas; se estimó el porcentaje de cobertura de cada especie de liquen; se determinaron los parámetros del micrositio ( $\mathrm{pH}$ de la corteza y diámetro a la altura del pecho, DAP) y del microclima (temperatura, humedad y radiación); se calculó la riqueza y la diversidad alfa y se efectuaron análisis multivariados para establecer las relaciones de forófito y líquen y de liquen y condición ambiental. Se registraron 21 especies, agrupadas en ocho familias y 14 géneros; la familia con mayor cobertura fue Arthoniaceae y la de mayor riqueza, Physciaceae; la especie más abundante fue Bacidia sp. El forófito Trichilia pallida presentó la riqueza y la diversidad más altas, en tanto que Cecropia angustifolia presentó la especie de liquen más dominante. Las especies de líquenes presentaron preferencia por un solo forófito, excepto Leptogium diaphanum, la cual se registró en dos forófitos. Los parámetros de $\mathrm{pH}$, intensidad lumínica, temperatura y DAP se correlacionaron positivamente con el establecimiento de los líquenes, los cuales registraron una diversidad significativa con el beneficio que ello conlleva para la conservación y el estudio de estos ecosistemas estratégicos de la región andina colombiana.
\end{abstract}

Palabras clave: líquenes; forófitos; microclimáticos; micrositio; diversidad.

\begin{abstract}
Our study aimed at contributing to the knowledge of the composition and diversity, phorophyte specificity, and microenvironmental preferences of corticolous lichens from a forest in the sub-Andean Centro region of Colombia (Ibague-Tolima). We selected 25 individuals from five tree species. We estimated the coverage percentage of each species of lichen and identified the microsite parameters (bark $\mathrm{pH}$ and diameter at breast height, $\mathrm{DBH}$ ), as well as the microclimatic variables (temperature, humidity, and radiation). We estimated the richness and the alpha diversity and we applied multivariate analysis to establish the phorophyte-lichen and lichen-environmental condition relationships. We recorded 21 species grouped in eight families and 14 genera. Arthoniaceae was the family with the greatest coverage and Physciaceae the one with the greatest richness. The most abundant species was Bacidia sp. Trichilia pallida phorophyte had the highest richness and diversity, while Cecropia angustifolia presented the most dominant species of lichen. Except Leptogium diaphanum, which was found in two phorophytes, the other species preferred one single phorophyte. The $\mathrm{pH}$, light intensity, temperature, and DAP were positively correlated to the establishment of lichens. We registered a significant diversity that opens the way to new research useful for preserving these strategic ecosystems of the Andean region in Colombia.
\end{abstract}

Keywords: Lichens; phorophytes; microclimatic; microsite; diversity.

*Correspondencia:

Alfredo Torres-Benítez; alfredo.torres@unibague.edu.co; Recibido: 3 de mayo de 2019; Aceptado: 9 de octubre de 2019; Editor: Elizabeth Castañeda 


\section{Introducción}

Los líquenes son una asociación simbiótica entre un hongo (denominado micobionte, el cual puede ser un ascomiceto o un basidiomiceto) y un organismo fotosintético (fotobionte, el cual frecuentemente es un alga verde o una cianobacteria) (Nash, 2008). También pueden formar simbiosis tripartitas o simbiodemas (hongo, alga verde y cianobacteria), que amplían el espectro de distribución y de crecimiento de los hongos liquenizados (Green \& Lange, 1991; Dahlman \& Palmqvist, 2003; Henskens, et al., 2012), como sucede en los géneros Lobaria, Pseudocyphellaria y Sticta de la familia Lobariaceae (Moncada, et al., 2013, 2014, 2015). La relación directa entre los líquenes y el ambiente mantiene un flujo constante de agua, carbono, nitrógeno y otros elementos esenciales que permite categorizarlos como bioindicadores, los cuales reaccionan ante los distintos niveles de fragmentación o alteración del medio (Lücking, et al., 2008, 2017), la contaminación y los cambios climáticos (Estrabou, 2007; Lijteroff, et al., 2009; Ardila, et al., 2014), y aportan información sobre los principales factores ambientales como el $\mathrm{pH}$, la disponibilidad de nutrientes, la humedad, la temperatura y la iluminación en sistemas maduros (Hernández et al., 1998; Lücking, 1999; Rosabal, et al., 2012b, 2013). Los líquenes cortícolas, también conocidos como líquenes epífitos, son aquellos que viven sobre un árbol que les sirve de sustrato (Chaparro \& Aguirre, 2002), les proporciona múltiples microhábitats y permite la colonización de líquenes foliosos, fruticosos, crustáceos epifleódicos y crustáceos endofleódicos (Aguirre, 2008; Rincón, et al., 2011). En cuanto a las especies epífitascortícolas en Colombia, las familias más diversas son Parmeliaceae, Physciaceae, Lobariaceae y Collemataceae, y entre los géneros más ricos en especies se encuentran Hypotrachyna, Leptogium, Sticta y Heterodermia (Aguirre, 2008).

En estudios locales en el departamento del Tolima (región Centro de Colombia), se han reportado comunidades abundantes de líquenes cortícolas con predominio de los géneros Parmotrema, Cladia, Stereocaulon y Acarospora (Restrepo \& Esquivel, 1996) y una mayor diversidad en la formación vegetal subtropical o premontana debido a las condiciones óptimas de temperatura y precipitación (Esquivel \& Nieto, 2003). En la zona suroccidental de Colombia la diversidad de líquenes cortícolas está representada principalmente por los géneros Herpothallon, Pyrenula, Graphis, Arthonia, Coenogonium y Phaeographis y las familias Arthoniaceae, Graphidaceae, Thelotremataceae y Pyrenulaceae (Soto \& Bolaños, 2010). Según un estudio sobre la especificidad de forófito y las preferencias microambientales, estas comunidades se relacionan con factores como la intensidad de la luz, la temperatura y el DAP y la ausencia de preferencia por un forófito (Soto, et al., 2012). Por otro lado, en la región biogeográfica del Chocó la diversidad y riqueza de líquenes cortícolas es alta (77 especies) y se relacionó con el tipo de bosque (húmedo o seco) y las condiciones del micrositio de los árboles hospederos (Soto, et al., 2015).

En otros estudios de zonas tropicales como Cuba, se han registrado hasta 115 especies de líquenes cortícolas, con una clara distribución vertical sobre los forófitos y en relación directa con las condiciones de luz, humedad y temperatura (Rosabal, et al., 2012). En zonas de Brasil se han reportado 150 especies de líquenes cortícolas denominadas raras, las cuales se relacionan medianamente con factores ambientales como la corteza del forófito, el microclima y la influencia de eventos aleatorios que afectan la dispersión de las especies (Cáceres, et al., 2007), así como con los cambios abruptos en el paisaje debido al reemplazo de especies hospederas nativas (Käffer, et al., 2009). En este contexto, y dada la necesidad de ampliar los estudios ecológicos que evidencien la interacción de los líquenes con su medio, el objetivo del presente trabajo fue caracterizar la diversidad, la especificidad de forófito y las preferencias microambientales de la comunidad de líquenes cortícolas en un bosque subandino de la región Centro de Colombia.

\section{Materiales y métodos}

Área de estudio. La zona de estudio correspondió al remanente de bosque subandino "El Espejo" ubicado a $1.120 \mathrm{~m}$ s.n.m. en el área de protección ambiental de la subcuenca del río Chipalo, en la zona urbana del municipio de Ibagué, Colombia (Figura 1).

Muestreo. Se seleccionaron cinco individuos maduros (Gradstein, et al., 2003) de cada una de las cinco especies arbóreas (forófitos) más abundantes en el remanente de bosque: Anacardium excelsum (Bertero ex Kunth) Skeels (caracolí), Trichilia pallida Sw. (cedro macho), Cecropia angustifolia Trécul (yarumo), Guarea guidonia (L.) Sleumer (Bilivil) y Tetrorchidium rubrivenium Poepp. (arenillo), para un total de 25 unidades de muestreo. En el estrato uno de cada forófito según la zonificación planteada por Johansson (1974), se ubicó un cuadrante de 50x20 cm a 1,3 m de altura desde la base en el lado oriental del tronco (Soto, et al., 2012) para estimar la abundancia relativa de cada especie de liquen.

Se evaluaron las preferencias microclimáticas de humedad relativa, temperatura ambiente (termohigrómetro) e irradiancia, con dos mediciones en el día (una en la mañana y otra en la tarde) utilizando un sensor de luz Hydrofarm ${ }^{\circledR}$ (LGBQM), así como preferencias del micrositio correspondientes al DAP y el $\mathrm{pH}$ de la corteza.

Recolección e identificación de especies. Los ejemplares de líquenes se depositaron en bolsas de papel y se secaron a temperatura ambiente; se hizo la determinación taxonómica mediante claves especializadas de líquenes neotropicales (Esquivel \& Nieto, 2003; Sipman, 2006). Para corroborar las determinaciones, el material liquénico fue revisado por profesionales del Grupo Colombiano de Liquenología.

Análisis de datos. En el análisis de datos se calculó la abundancia y la composición a nivel de familia, género 


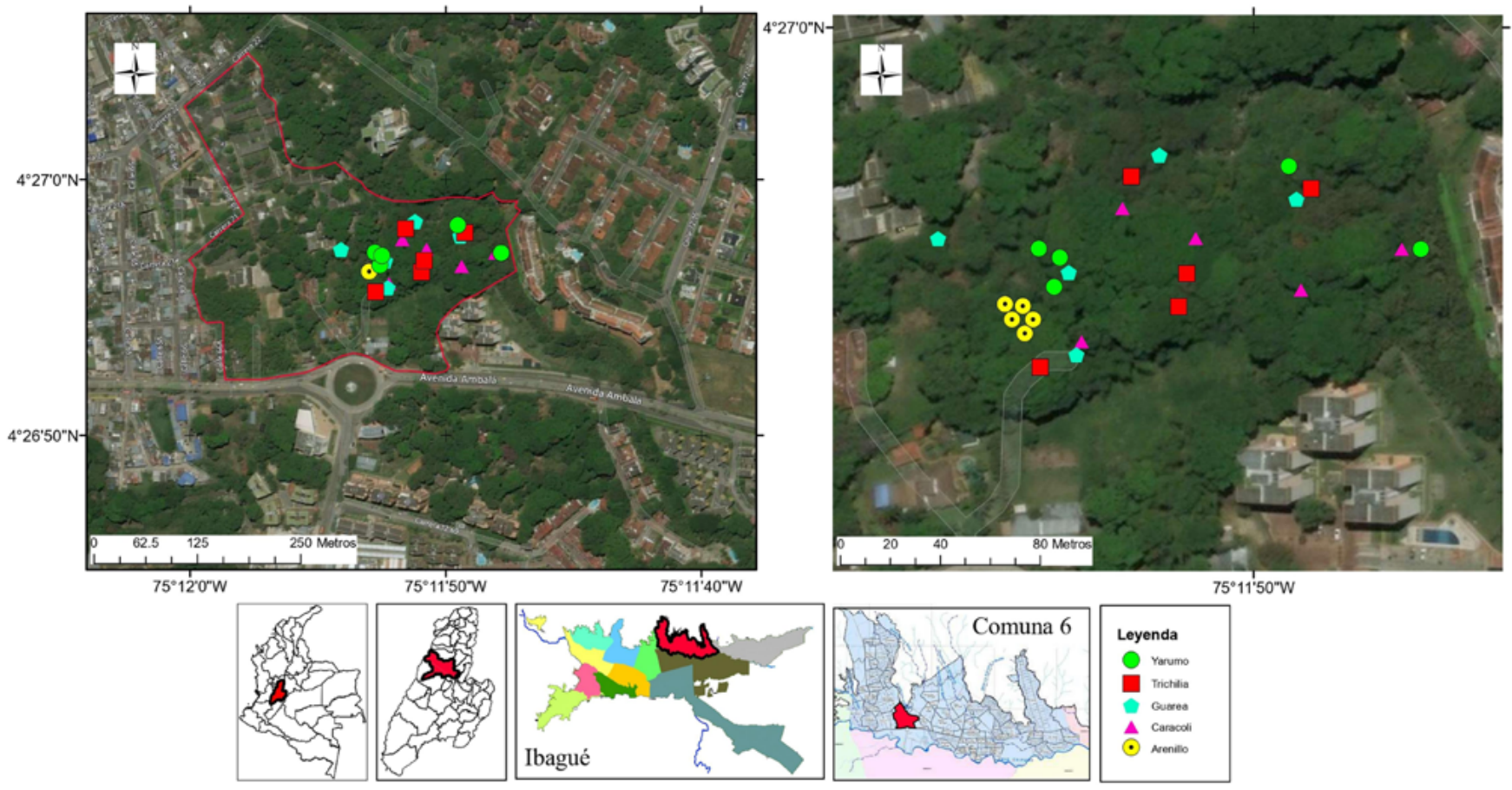

Figura 1. Distribución de los forófitos seleccionados en el remanente de bosque El Espejo

y especie. Los índices de riqueza y diversidad (Margalef, Shannon-Weaver y dominancia) se calcularon con el programa estadístico Infostat (Casanoves, et al., 2012); además, se hizo una curva de acumulación de especies con los estimadores Chao 1, Chao 2, Jacknife 1, Jacknife 2 y bootstrap para cada uno de los forófitos estudiados. Para la evaluación de la asociación entre las especies de líquenes cortícolas y forófitos se hizo un análisis de correspondencia simple (ACS) y la prueba de ji al cuadrado mediante la combinación de ejes necesarios para explicar más del $52,8 \%$ de la variabilidad de los datos; además, se hizo escalamiento multidimensional no métrico (non-metric multidimensional scaling, NMDS). Para establecer la asociación de la comunidad liquénica con las condiciones microclimáticas y del micrositio se hizo un análisis de componentes principales (ACP).

\section{Resultados}

Diversidad de líquenes cortícolas. La representatividad general de las especies fluctuó entre 77 y $94 \%$, lo cual indica una riqueza esperada de 21 a 26 especies para el área de estudio (Figura 2), sin incluir otras especies de árboles. La mayor representatividad se evidenció en T. pallida y G. guidonia (100\%), seguidos de $T$. rubrivenium y $C$. angustifolia (73-92\%) у A. excelsum (68-92\%).

En los 25 forófitos seleccionados se registraron 21 especies de líquenes distribuidas en ocho familias y 14 géneros (Tabla 1). El $84 \%$ de la cobertura liquénica correspondió a las familias Arthoniaceae (37\%), Ramalinaceae (25\%) y Collemataceae $(22 \%)$. Las familias con mayor riqueza fueron Physciaceae (seis especies), Arthoniaceae (cinco especies) y Collemataceae (tres especies). Las especies con mayor abundancia fueron Bacidia sp. (28 \%), Cryptothecia striata $\mathrm{G}$. Thor (18\%), Leptogium diaphanum (Sw.) Mont. (14\%), Herpothallon sp.2 (13\%), Cryptothecia sp.1 (7\%), Physcia sp.1 (6\%) y Leptogium submarginellum I.P.R. Cunha \& M.P. Marcelli (3 \%), las cuales representaron el $89 \%$ de la cobertura registrada.

El índice de dominancia de la comunidad liquénica fue alto $(0,62)$, en tanto que el índice de riqueza de Margalef fue medio-bajo $(0,33)$ y el índice de diversidad de ShannonWeaver, alto $(0,67)$. El forófito T. pallida obtuvo el promedio más alto del índice de diversidad $(0,78)$ y el forófito $C$. angustifolia presentó el promedio de diversidad más bajo $(0,15)$; en cuanto al índice de dominancia, C. angustifolia obtuvo el mayor promedio $(0,95)$ y $T$. pallida presentó el promedio más bajo $(0,50)$; para el índice de riqueza, $T$. pallida obtuvo el promedio más alto $(0,53)$ y $T$. rubrivenium, el más bajo $(0,21)$.

Especificidad de forófitos. Según el análisis de correspondencia simple, los dos primeros ejes explicaron el $52,8 \%$ de la variación en los datos, lo cual evidenció una asociación entre las especies de líquenes cortícolas y las especies de forófitos (Figura 3). Las especies L. submarginellum, Heterodermia japonica (M. Satô) Swinscow \& Krog, Cryptothecia sp.2, y Cryptothecia sp.1 se encontraron fuertemente asociadas con el forófito T.pallida. L. diaphanum, Physcia alba (Feé) Müll. Arg., Physcia crispa Nyl, Bacidia sp.1, y Phyllopsora sp.1 se asociaron con el forófito $C$. angustifolia, en tanto que Parmotrema bangii (Vain.) Hale, Opegrapha sp.1, Herpothallon sp.2 y L. diaphanum se 

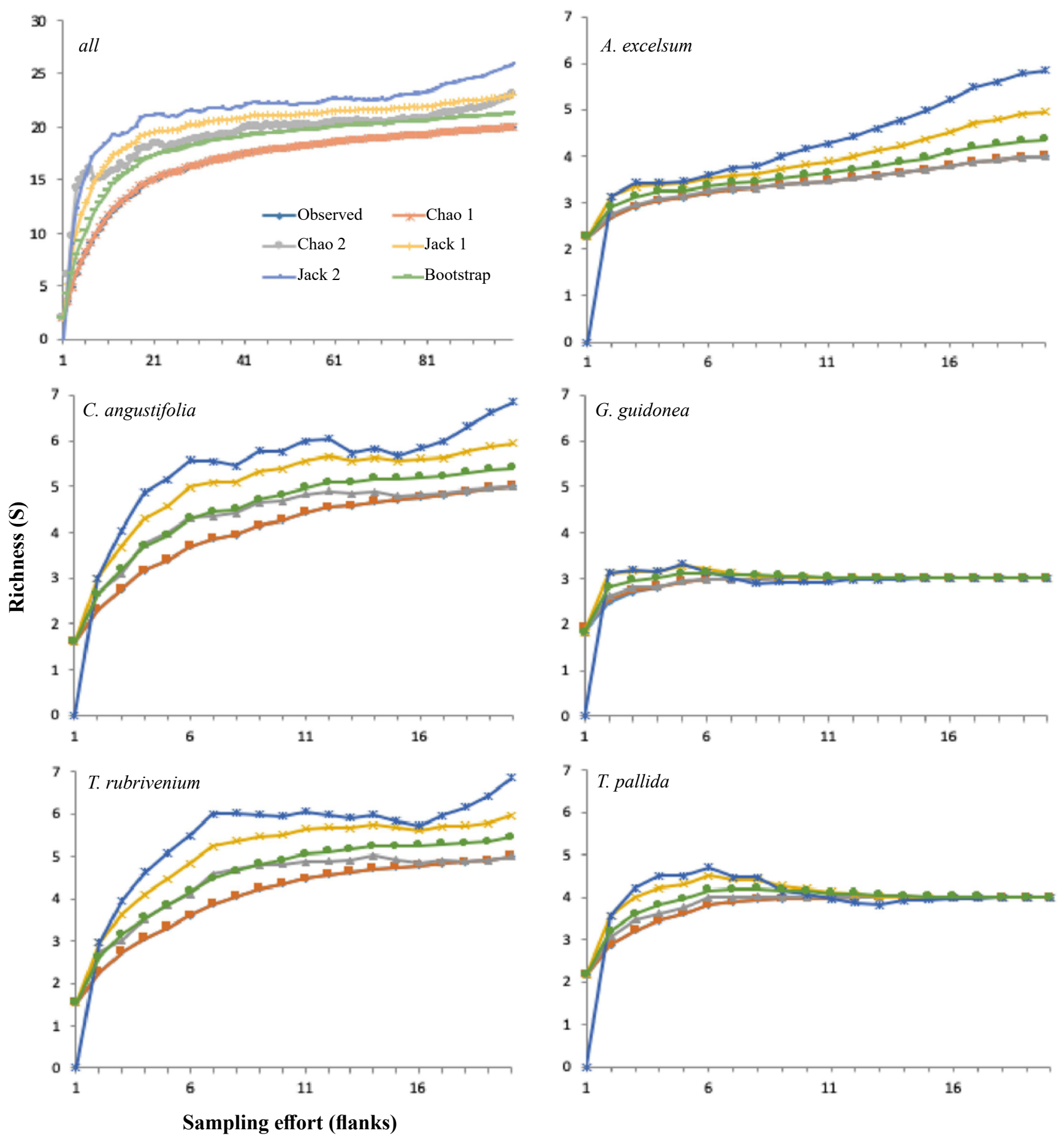

Figura 2. Curvas de acumulación de las especies de forófitos

asociaron con el forófito G. guidonia. Así mismo, Porina sp.1, Physcia sp.1, Physcia aipolia (Ehrh. ex Humb.) Fürnr., Pyrenula sp.1 y Opegrapha sp.2 presentaron una asociación con el T. rubrivenium. Por último, las especies C. striata, Physcia tenuis Moberg, Herpothallon sp.1 y Leptogium azureum (Sw. ex Ach.) Mont. se asociaron con el forófito $A$. excelsum.
El NMDS y el análisis de similitud (ANOSIM) evidenciaron una diferencia significativa en la estructura de los ensambles liquénicos asociados con los forófitos T. pallida, A. excelsum y $T$. rubrivenium $(\mathrm{r}=0,99$ y $\mathrm{p}=0,001)$. Sin embargo, en $C$. angustifolia y $G$. guidonia se evidenció la presencia de una especie de liquen en común (L. diaphanum) (Figura 4). 
Parámetros microclimáticos y del micrositio. Los individuos del forófito $A$. excelsum obtuvieron el promedio más alto de DAP $(105,03 \mathrm{~cm})$ y el más bajo se registró en los

Tabla 1. Especies de líquenes cortícolas reportadas en el remanente de bosque El Espejo

\begin{tabular}{ll}
\hline Familia & Especie \\
\hline Arthoniaceae & Cryptothecia sp. 1 \\
\hline Arthoniaceae & Cryptothecia sp. 2 \\
\hline Arthoniaceae & Cryptothecia striata \\
\hline Arthoniaceae & Herpothallon sp.1 \\
\hline Arthoniaceae & Herpothallon sp. 2 \\
\hline Collemataceae & Leptogium azureum \\
\hline Collemataceae & Leptogium diaphanum \\
\hline Collemataceae & Leptogium submarginelum \\
\hline Parmeliaceae & Parmotrema bangii \\
\hline Physciaceae & Heterodermia japónica \\
\hline Physciaceae & Physcia aipolia \\
\hline Physciaceae & Physcia alba \\
\hline Physciaceae & Physcia crispa \\
\hline Physciaceae & Physcia sp.1 \\
\hline Physciaceae & Physcia tenuis \\
\hline Porinaceae & Porina sp.1 \\
\hline Pyrenulaceae & Pyrenula sp.1 \\
\hline Ramalinaceae & Bacidia sp.1 \\
\hline Ramalinaceae & Phyllopsora sp.1 \\
\hline Roccellaceae & Opegrapha sp.1 \\
\hline Roccellaceae & Opegrapha sp.2 \\
\hline
\end{tabular}

individuos de T. rubrivenium $(10,63 \mathrm{~cm})$. En cuanto al $\mathrm{pH}$ de la corteza, el promedio más bajo fue para los individuos del forófito G. guidonia $(5,00)$ y el más alto para los individuos del forófito $C$. angustifolia $(7,94)$; el promedio más alto de intensidad de la luz se registró en los individuos del forófito C. angustifolia $\left(13,48 \mathrm{~W} / \mathrm{m}^{2}\right)$ y el más bajo, en los de $T$. rubrivenium $\left(0,84 \mathrm{~W} / \mathrm{m}^{2}\right)$. En cuanto a la humedad relativa, los individuos del forófito T. rubrivenium obtuvieron el promedio más alto $(75,84 \%)$ y el más bajo fue para los individuos de $C$. angustifolia $(45,31 \%)$. Por último, los individuos del forófito $G$ guidonia registraron el promedio más alto de temperatura $\left(29,53{ }^{\circ} \mathrm{C}\right)$ y los individuos del forófito T. rubrivenium, el más bajo $\left(23,75^{\circ} \mathrm{C}\right)$ (Tabla 2$)$.

Para las preferencias microambientales, el análisis de componentes principales en los dos primeros factores explicó el 59,3\% de la variabilidad. Las especies de líquenes Opegrapha sp.1, Phyllopsora sp.1, P. alba, Bacidia sp.1 y $L$. diaphanum se relacionaron con los forófitos de $\mathrm{pH}$ básico; P. crispa, P. bangii y Herphotallon sp. 2 se asociaron con una temperatura promedio de $29,25^{\circ} \mathrm{C}$, y una intensidad lumínica promedio de $1,74 \mathrm{~W} / \mathrm{m}^{2}$; por otro lado, $L$. submarginellum y $H$. japonica se relacionaron con un DAP promedio de $56,02 \mathrm{~cm}$; además, las especies liquénicas P. aipolia, Porina sp.1, Opegrapha sp.2, Physcia sp.1. y Pyrenula sp.1, no presentaron relación con el DAP, puesto que su desarrollo no se encontró ligado a fustes gruesos (Figura 5).

Todos los parámetros microambientales fueron consistentes y aportaron información significativa para el análisis, sin embargo, no se presentaron agrupamientos significativos entre las variables. El pH, la intensidad lumínica, la temperatura y el DAP se correlacionaron positivamente, mientras que la humedad relativa se correlacionó negativamente con las demás variables.

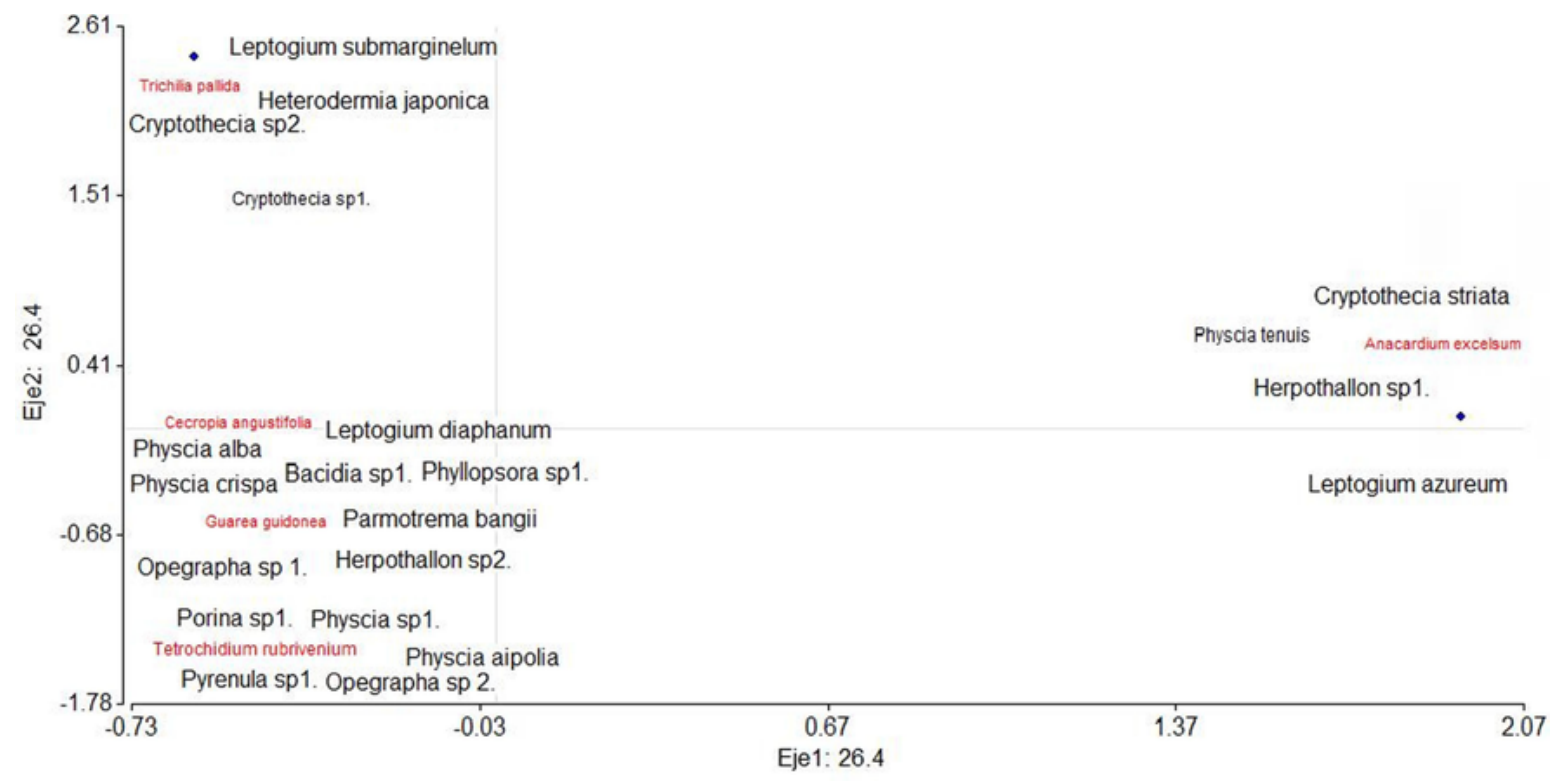

Figura 3. Análisis de correspondencia simple entre forófitos y especies de líquenes 


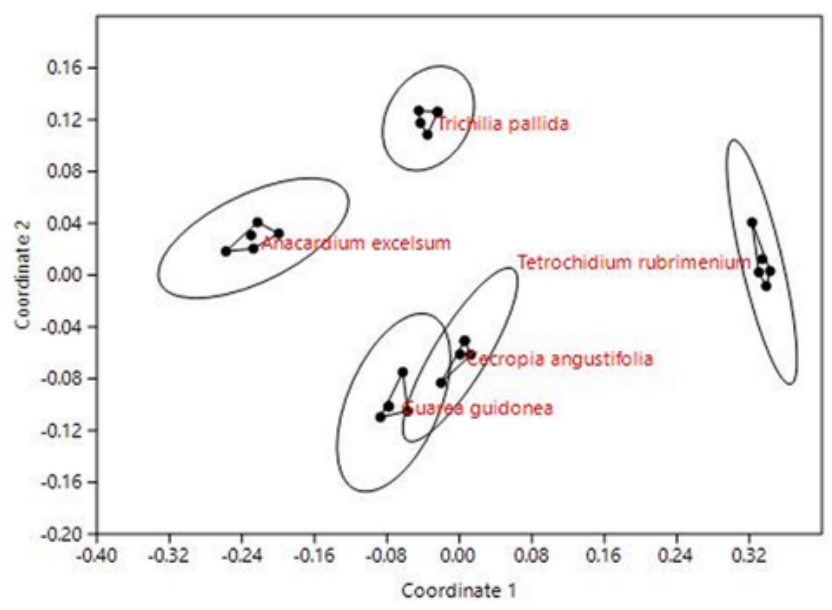

Figura 4. Análisis de escalamiento multidimensional no métrico de la relación entre forófitos y especies de líquenes

\section{Discusión}

La riqueza de especies del bosque El Espejo, representada en 21 especies, se puede considerar baja según los estudios realizados por Wolf (1993), Komposch \& Hafellner (2000), Cáceres \& Lücking (2007), Soto \& Bolaños (2010) y Soto, et al. (2012), pero, las unidades de muestreo en estos estudios fueron mayores. En términos generales, el conocimiento de la diversidad de los líquenes epífitos proporciona evidencia sustantiva para la conservación a escala de paisaje (Li, et al., 2013; Estrabou, et al., 2014). Por su parte, Physciaceae y Arthoniaceae, las familias con mayor abundancia y diversidad de especies en el bosque, poseen una amplia distribución geográfica y alta tolerancia frente a las perturbaciones (Soto \& Bolaños, 2010; Ochoa, et al., 2015), y colonizan en una amplia variedad de formaciones vegetales en el Trópico (Herrera, et al., 2014). Asimismo,

Tabla 2. Riqueza y parámetros microambientales asociados a los forófitos en el remanente de bosque El Espejo

\begin{tabular}{|c|c|c|c|c|c|c|}
\hline Forófito & $\mathbf{S}$ & pH & DAP (cm) & IL & HR\% & Temperatura $\left({ }^{\circ} \mathrm{C}\right)$ \\
\hline A. excelsum 1 & 3 & 5,90 & 122,55 & 15,10 & 72,35 & 26,30 \\
\hline A. excelsum 2 & 3 & 6,40 & 62,71 & 2,69 & 72,25 & 26,95 \\
\hline A. excelsum 3 & 3 & 6,00 & 125,10 & 1,62 & 77,75 & 26,30 \\
\hline A. excelsum 4 & 3 & 6,40 & 118,73 & 6,74 & 73,85 & 26,05 \\
\hline A. excelsum 5 & 4 & 5,90 & 97,40 & 2,09 & 72,05 & 26,05 \\
\hline Promedio \pm DE & $3,2 \pm 0,45$ & $6,12 \pm 0,26$ & $105,30 \pm 26,20$ & $5,65 \pm 5,66$ & $73,65 \pm 2,40$ & $26,33 \pm 0,37$ \\
\hline G. guidonia 1 & 3 & 5,40 & 25,78 & 1,72 & 47,50 & 30,55 \\
\hline G. guidonia 2 & 3 & 4,20 & 42,97 & 38,90 & 48,50 & 29,80 \\
\hline G. guidonia 3 & 3 & 5,40 & 30,24 & 1,90 & 51,25 & 29,25 \\
\hline G. guidonia 4 & 3 & 4,50 & 46,15 & 1,44 & 50,35 & 28,85 \\
\hline G. guidonia 5 & 1 & 5,50 & 58,89 & 2,23 & 47,90 & 29,20 \\
\hline Promedio $\pm \mathrm{DE}$ & $2,60 \pm 0,89$ & $5,00 \pm 0,60$ & $40,81 \pm 13,21$ & $9,24 \pm 16,59$ & $49,10 \pm 1,62$ & $29,53 \pm 0,66$ \\
\hline T. pallida 1 & 4 & 6,30 & 37,56 & 0,73 & 53,65 & 28,40 \\
\hline T. pallida 2 & 4 & 6,40 & 106,63 & 16,68 & 53,35 & 28,80 \\
\hline T. pallida 3 & 2 & 6,10 & 56,02 & 1,14 & 56,65 & 28,05 \\
\hline T. pallida 4 & 4 & 7,40 & 45,52 & 2,75 & 56,45 & 28,55 \\
\hline T. pallida 5 & 4 & 5,30 & 79,90 & 2,06 & 58,20 & 28,45 \\
\hline Promedio \pm DE & $3,60 \pm 0,89$ & $6,30 \pm 0,75$ & $65,13 \pm 28,14$ & $4,67 \pm 6,75$ & $55,66 \pm 2,09$ & $28,45 \pm 0,27$ \\
\hline T. rurbivenium 1 & 2 & 6,60 & 10,82 & 1,21 & 74,65 & 24,40 \\
\hline T. rurbivenium 2 & 2 & 6,10 & 7,32 & 0,64 & 76,15 & 23,75 \\
\hline T. rurbivenium 3 & 3 & 7,10 & 10,82 & 1,10 & 76,40 & 23,75 \\
\hline T. rurbivenium 4 & 2 & 6,50 & 13,05 & 0,55 & 77,55 & 23,15 \\
\hline T. rurbivenium 5 & 3 & 6,40 & 11,14 & 0,72 & 74,45 & 23,70 \\
\hline Promedio $\pm \mathrm{DE}$ & $2,40 \pm 0,55$ & $6,54 \pm 0,36$ & $10,63 \pm 2,07$ & $0,84 \pm 0,29$ & $75,84 \pm 1,29$ & $23,75 \pm 0,44$ \\
\hline C. angustifolia 1 & 3 & 7,80 & 31,19 & 57,04 & 44,65 & 29,85 \\
\hline C. angustifolia 2 & 4 & 7,40 & 37,56 & 1,15 & 46,05 & 29,60 \\
\hline C. angustifolia 3 & 3 & 9,30 & 37,56 & 2,42 & 39,55 & 28,55 \\
\hline C. angustifolia 4 & 2 & 7,70 & 23,24 & 5,65 & 49,70 & 28,60 \\
\hline C. angustifolia 5 & 2 & 7,50 & 27,06 & 1,13 & 46,60 & 28,85 \\
\hline Promedio $\pm \mathrm{DE}$ & $2,80 \pm 0,84$ & $7,94 \pm 0,78$ & $31,32 \pm 6,35$ & $13,48 \pm 24,42$ & $45,31 \pm 3,71$ & $29,09 \pm 0,60$ \\
\hline
\end{tabular}

DE: desviación estándar; S: riqueza; DAP: diámetro a la altura del pecho $(\mathrm{cm})$; IL: intensidad de luz; HR: humedad relativa 


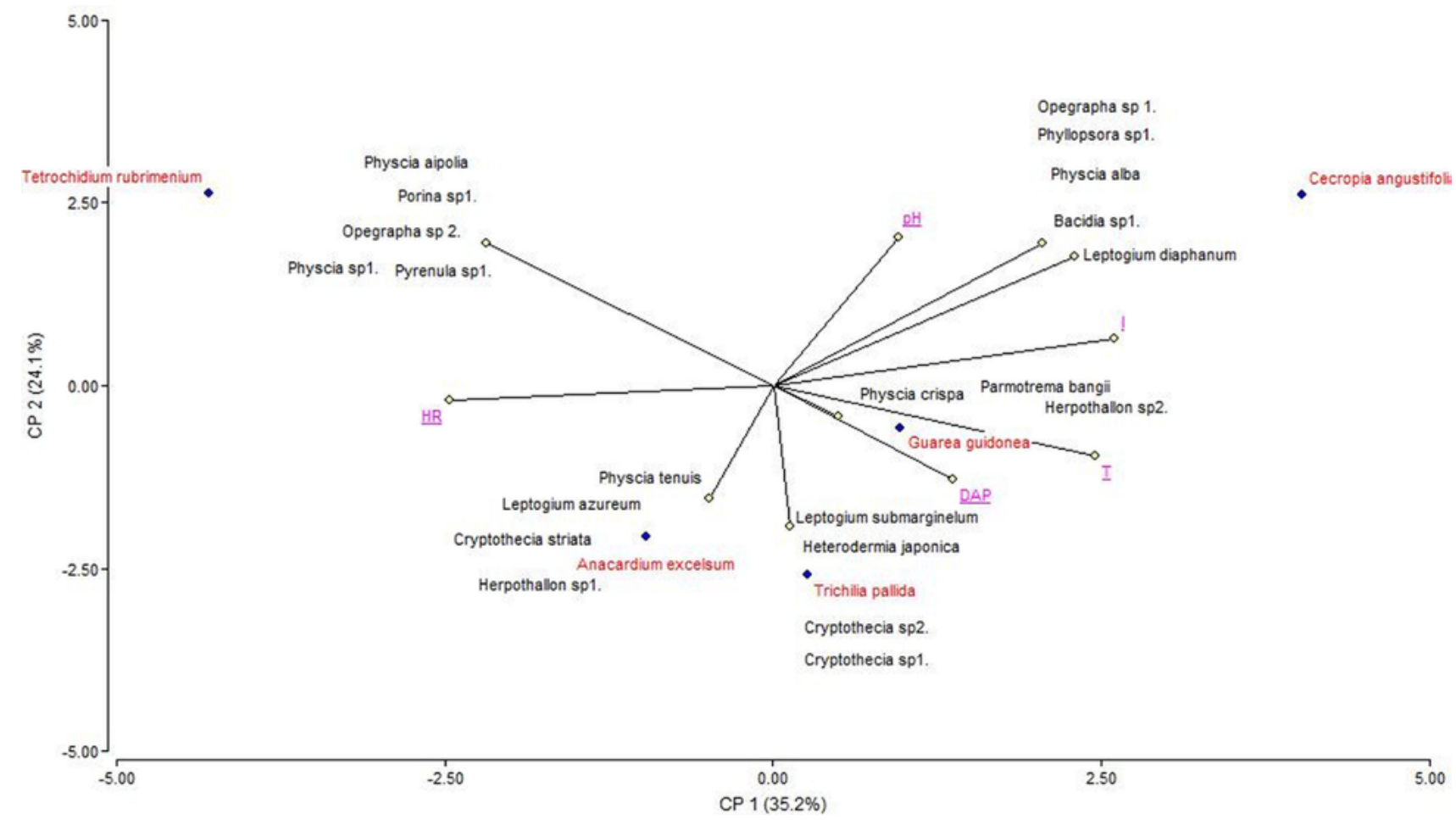

Figura 5. Análisis de componentes principales de la relación entre variables microambientales y especies de líquenes

la familia Parmeliaceae hace parte de la diversidad liquénica del bosque y se ha reportado en un amplio rango de hábitats con abundantes géneros y especies (Leavitt, et al., 2015) en el Neotrópico (Rincón, et al., 2011; Rosabal, et al., 2012a), especialmente en las formaciones vegetales andinas y subandinas (Aguirre, 2008).

El remanente de bosque presenta un ensamble de especies liquénicas particular, donde se destacan Bacidia sp., $C$. striata, L. diaphanum, Herpothallon sp.2 y Cryptothecia sp.1, lo que contrasta con los hallado en estudios locales en la zona urbana y periurbana de la ciudad de Ibagué (Tolima, Colombia), en los cuales se registra la abundancia de las especies Physcia undulata Moberg., P. aipolia, Candelaria concolor (Dicks.) Arnold., Physcia lacinulata Müll.Arg. y Pyxine pyxinoides (Müll.Arg.) Kalb. (Moreno, et al., en preparación). La mayoría de estas comunidades se caracterizan por tener un talo costroso y folioso, que constituyen las formas de crecimiento más comunes en Colombia (Soto, et al., 2010; Rincón, et al., 2011). Además, debido a la heterogeneidad ambiental de la zona andina colombiana, se conforman microhábitats que diversifican las comunidades de líquenes cortícolas (Grandstein, et al., 1996) y generan el establecimiento de biotipos útiles para la bioindicación del estado de perturbación o conservación de un bosque (Ramírez, et al., 2016).

Las especies de líquenes del bosque El Espejo muestran una alta especificidad por el forófito, dado que las especies arbóreas seleccionadas en el área de estudio son las de mayor dominancia en la comunidad vegetal. Esta condición difiere de los resultados obtenidos por Cáceres \& Lücking (2007) y Soto, et al. (2012), en cuyos estudios se reporta una limitada asociación directa entre líquen y forófito en áreas con gran diversidad de árboles con una mayor oferta de sustratos y menos probabilidad de preferencia. El forófito $A$. excelsum presentó la mayor especificidad de especies liquénicas, sin embargo, es probable que al ampliar el muestreo en los demás flancos y en los diferentes estratos verticales, se evidencien especies compartidas con los demás forófitos.

Por otro lado, el agrupamiento de las especies de líquenes en el bosque se encuentra influenciado por los factores microambientales que aumentan significativamente su diversidad (Cáceres, et al., 2007; Soto, et al., 2012,), especialmente por las variables del $\mathrm{pH}$, el DAP, la temperatura y la intensidad lumínica, que, según Wolf (1993a,b), Holz (2003), Soto, et al. (2012) y Simijaca, et al. (2018), afectan el establecimiento de las comunidades liquénicas en los forófitos y se relacionan directamente con el desarrollo de rasgos funcionales como el tipo de fotobionte, la forma de crecimiento, la estructura reproductiva y el contenido de metabolitos secundarios (Mossmann, et al., 2013; Benítez et al., 2018).

\section{Conclusiones}

El remanente de bosque subandino "El Espejo" presenta una significativa diversidad liquénica con preferencias específicas por forófitos. Las condiciones de $\mathrm{pH}$ ácido, de la penetración de luz media, de diámetro amplio y de temperatura superior a $28{ }^{\circ} \mathrm{C}$, son determinantes para el establecimiento de la 
especies de líquenes, en tanto que la humedad relativa no representa una variable ambiental que influya directamente en el ensamblaje de la comunidad liquénica del bosque.

\section{Contribución de los autores}

Los tres autores participaron en el diseño, ejecución y análisis de los datos, así como en la elaboración y ajuste del manuscrito.

\section{Conflicto de intereses}

Los autores declaran no tener conflicto de intereses.

\section{Agradecimientos}

Esta investigación fue financiada por el Convenio entre la Universidad de Ibagué y la Gobernación del Tolima, código 17-484-SEM.

\section{Referencias}

Aguirre, J. (2008). Diversidad y Riqueza de los líquenes en la región natural andina o sistema cordillerano. En O. Rangel. (Ed.), Colombia Diversidad Biótica VI Riqueza y diversidad de los musgos y líquenes en Colombia. Bogotá D.C., Colombia: Editorial Universidad Nacional de Colombia. p. 337-382.

Ardila, A., Moncada, B., Lücking, R. (2014). Epiphyte homogenization and de-diversification on alien Eucalyptus versus native Quercus forest in the Colombian Andes: A case study using lirellate Graphidaceae lichens. Biodiversity and Conservation. 24: 1239-1252. Doi: 10.1007/s10531014-0855-7

Benítez, A., Aragón, G., González, Y., Prieto, M. (2018). Functional traits of epiphytic lichens in response to forest disturbance and as predictors of total richness and diversity. Ecological Indicators. 86: 18-26. Doi: 10.1016/j. ecolind.2017.12.021

Cáceres, M., Lücking, R., Rambold, G. (2007). Phorophyte specificity and environmental parameters versus stochasticity as determinants for species composition of corticolous crustose lichen communities in the Atlantic rain forest of northeastern Brazil. Mycological Progress. 6: 117-136. Doi: 10.1007/s11557-007-0532-2

Casanoves, F., Balzarini, M.G., Di Rienzo, J.A., González, L., Tablada, E., Robledo, C.W. (2012). InfoStat, versión 2012. Manual de Usuario. Grupo InfoStat, FCA, Universidad Nacional de Córdoba. Córdoba, Argentina. Editorial Brujas Argentina.

Chaparro, M. \& Aguirre, J. (2002). Hongos liquenizados. Bogotá D.C., Colombia. Editorial Universidad Nacional de Colombia. 220 pp.

Dahlman, L. \& Palmqvist, K. (2003). Growth in two foliose tripartite lichens, Nephroma arcticum and Peltigera aphthosa: Empirical modeling of external vs internal factors. Functional Ecology. 17: 821-831. Doi: 10.1046/j.02698463.2003.00804.x

Esquivel, H. \& Nieto, A. (2003). Diversidad florística de la cuenca alta del río Combeima. Ibagué, Colombia. Editorial León Gráficas. 190 pp.

Estrabou, C. (2007). Preferencias de forófito por los líquenes en el bosque chaqueño oriental. Bosque. 28: 46-49. Doi: 10.4067/S0717-92002007000100007
Estrabou, C., Quiroga, C., Rodríguez, J.M. (2014). Lichen community diversity on a remnant forest in south of Chaco region (Córdoba, Argentina). Bosque. 35: 49-55. Doi: 10.4067/S0717-92002014000100005

Gradstein, R., Hietz, P., Lücking, R., Luecking, A., Sipman, H., Vester, H., Wolf, J., Gardette, E. (1996). How to sample the epiphytic diversity of tropical rain forests. Ecotropica. 2: 59- 72.

Gradstein, S.R., Nadkarni, N.M., Krömer, T., Holz, I., Nöske, N. (2003). A protocol for rapid and representative sampling of vascular and non-vascular epiphyte diversity of tropical rain forest. Selbyana. 24: 105-111. Doi: 10.2307/41750962

Green, T.G. \& Lange, O. (1991). Ecophysiological adaptations of the lichen genera Pseudocyphellaria and Sticta to south temperate rainforests. Lichenologist. 23: 267-282. Doi: 10.1017/S0024282991000427

Henskens, F., Green, T.G., Wilkins, A. (2012). Cyanolichens can have both cyanobacteria and green alga in a common layer as major contributors to photosynthesis. Annals of Botany. 110: $555-563$. Doi: $10.1093 / \mathrm{aob} / \mathrm{mcs} 108$

Hernández, Y., Díaz, A., Rowe, J. (1998). Distribución de los macrolíquenes corticícolas y su relación con la vegetación en el Parque Natural de los Alcornocales (Cádiz, S de España). Acta Botánica Malacitana. 23: 43-50.

Herrera, M., Lücking, R., Pérez, R.E., Miranda, R., Sánchez, N., Barcenas, A., Carrizosa, A., Zambrano, A., Ryan, B., Nash, T. (2014). Biodiversidad de líquenes en México. Revista Mexicana de Biodiversidad, Supl. 85: S82-S99. Doi: $10.7550 / \mathrm{rmb} .37003$

Holz, I. (2003). Diversidad y Ecología de las briófitas y líquenes macro en primaria y secundaria Bosques Montanos Quercus, Cordillera de Talamanca, Costa Rica. Tesis Doctorado en Ciencias. Gotinga, Alemania. Facultad de Ciencias, Universidad de Gotinga.

Johansson, D. (1974). Ecology of vascular epiphytes in west African rain forest. Acta Phytogeografica Suecica. 59: 1-136.

Käffer, M.I., Ganade, G., Marcelli, M.P. (2009). Lichen diversity and composition in Araucaria forests and tree monocultures in southern Brazil. Biodiversity and Conservation. 18: 3543-3561. Doi: 10.1007/s10531-009-9659-6

Komposch, H. \& Hafellner, J. (2000). Diversity and vertical distribution of lichens in a Venezuelan tropical lowland rain forest. Selbyana. 21: 11-24.

Leavitt, S.D., Kraichak, E., Nelsen, M.P., Altermann, S., Divakar, P.K., Alors, D., Esslinger, T.L., Crespo, A., Lumbsch, H.T. (2015). Fungal specificity and selectivity for algae play a major role in determining lichen partnerships across diverse ecogeographic regions in the lichenforming family Parmeliaceae (Ascomycota). Molecular Ecology. 24: 3779-3797. Doi: 10.1111/mec.13271

Li, S., Liu, W., Li, D. (2013). Epiphytic lichens in subtropical forest ecosystems in southwest China: Species diversity and implications for conservation. Biological Conservation. 159: 88-95. Doi: 10.1016/j.biocon.2012.12.027

Lijteroff, R., Lima, L., Prieri, B. (2009). Uso de líquenes como bioindicadores de contaminación atmosférica en la ciudad de San Luis, Argentina. Rev. Int. Contam. Ambient. 25: 111-120.

Lücking, R. (1999). Ecology of foliicolous lichens at the "Botarrama" trail (Costa Rica), a neotropical rain forest. I. Species composition and its ecogeographical implications. Biotropica. 31: 553-564. Doi: 10.1111/j.1744-7429.1999. tb00402.x 
Lücking, R., Aptroot, A., Chaves, J.L., Sipman, H.J.M., Umaña, L. (2008). A first assessment of the ticolichen biodiversity inventory in Costa Rica: The genus Graphis, with notes on the genus Hemithecium (Ascomycota: Ostropales: Graphidaceae). Fieldiana Botany. 46: 1-126. Doi: 10.3158/0015-0746(2008)46[1:AFAOTT]2.0.CO;2

Lücking, R., Dal Forno, M., Moncada, B., Coca, L.F., VargasMendoza, L.Y., Aptroot, A., et al. (2017). Turbotaxonomy to assemble a megadiverse lichen genus: seventy new species of Cora (Basidiomycota: Agaricales: Hygrophoraceae), honouring David Leslie Hawksworth's seventieth birthday. Fungal Diversity. 84: 139-207. Doi: 10.1007/s13225-016-0374-9

Moncada, B., Coca, L.F., Lücking, R. (2013). Neotropical members of Sticta (lichenized Ascomycota: Lobariaceae) forming photosymbiodemes, with the description of seven new species. The Bryologist. 116: 169-200. Doi: 10.1639/00072745-116.2.169

Moncada, B., Aguirre, J., Lücking, R. (2014). Ecogeografía del género Sticta (Ascomycota liquenizados: Lobariaceae) en Colombia. Revista Biología Tropical. 62: 257-272. Doi: 10.15517/RTB.V62I1.3564

Moncada, B., Súarez, A., Lücking, R. (2015). Nueve especies nuevas del género Sticta (Ascomycota liquenizados: Lobariaceae) del morfotipo fuliginosa sensu lato de Colombia. Revista de la Academia Colombiana de Ciencias Exactas, Físicas y Naturales. 39: 50-66. Doi: $10.18257 /$ raccefyn. 110

Mossmann, N., de Azevedo, S.M., Lucheta, F., Müller, S.C. (2013). Functional diversity and traits assembly patterns of lichens as indicators of successional stages in a tropical rainforest. Ecological Indicators. 34: 22-30. Doi: 10.1016/j. ecolind.2013.04.012

Nash, T. (2008). Lichen Biology. Cambridge, Gran Bretaña. Editorial Cambridge University.

Ochoa, D., Cueva, A., Prieto, M., Aragón, G., Benítez, A. (2015). Cambios en la composición de líquenes epífitos relacionados con la calidad del aire en la ciudad de Loja (Ecuador). Caldasia. 37: 333-343. Doi: 10.15446/Caldasia. v37n2.53867

Ramírez, N.A., León, M., Lücking, R. (2016). Uso de biotipos de líquenes como bioindicadores de perturbación en fragmentos de bosque altoandino (Reserva Biológica "Encenillo", Colombia). Caldasia. 38: 31-52. Doi: 10.15446/caldasia. v38n1.57821
Restrepo, R. \& Esquivel, H. (1996). Estudio taxonómico e importancia ecológica de los líquenes del jardín botánico Alejandro Von Humboldt de la Universidad del Tolima. Tesis Biólogo. Ibagué, Colombia. Facultad de Ciencias, Universidad del Tolima.

Rincón, A., Aguirre, J., Lücking, R. (2011). Líquenes corticícolas en el Caribe colombiano. Caldasia. 33: 331-374.

Rosabal, D., Burgaz, A., Reyes, O. (2012a). Diversidad y distribución vertical de líquenes corticícolas en la pluvisilva montana de la Gran Piedra, Cuba. Bot. Complut. 36: 19-30. Doi: 10.5209/rev_BOCM.2012.v36.39439

Rosabal, D., Burgaz, A.R., Altamirano, A., Aragón, G. (2012b). Differences in diversity of corticolous lichens between interior and edge of the Monte Barranca semi-deciduous forest, Santiago de Cuba. The Briologist. 115: 333-340. Doi: 10.1639/0007-2745-115.2.333

Rosabal, D. Burgaz, A.R., Reyes, O.J. (2013). Substrate preferences and phorophyte specificity of corticolous lichens on five tree species of the montane rainforest of Gran Piedra, Santiago de Cuba. The Bryologist. 116: 113-121. Doi: 10.1639/0007-2745-116.2.113

Simijaca, D., Moncada, B., Lucking, R. (2018). Bosque de roble o plantación de coníferas, ¿qué prefieren los líquenes epífitos? Colombia Forestal. 21: 123-141.

Soto, E. \& Bolaños, A. (2010). Diversidad de líquenes cortícolas en el bosque subandino de la finca Zíngara (Cali, valle del cauca). Revista de Ciencias. 14: 35-44.

Soto, E., Lucking, R., Bolaños, A. (2012). Especificidad de forofito y preferencias microambientales de los líquenes cortícolas en cinco forófitos del bosque premontano de la finca Zíngara, Cali, Colombia. Biología Tropical. 60: 843-856.

Soto, E., Londoño, V., Diaz, D. (2015). Epiphytes from a forest type transition zone in the Choco Biogeographic región, Valle del Cauca, Colombia. Biología Tropical. 63: 915-926.

Sipman, H.J. (2006). Lichen determination keys available on internet. Botanischer Garten und Botanisches Museum Berlin. Fecha de consulta: julio a diciembre de 2018. Disponible en: http://www.bgbm.org/Sipman/keys/default.htm

Wolf, J. (1993a). Diversity patterns and biomass of epiphytic bryophytes and lichens along an altitudinal gradient in the northern Andes. Annals of the Missouri Botanical Garden. 80: $928-960$. Doi: $10.2307 / 2399938$

Wolf, J. (1993b). Epiphyte communities of tropical montane rain forest in the northern Andes II. Uper montane communities. Phytocoenologia. 22: 53-103. Doi: 10.1127/phyto/22/1993/53 